\title{
Comportamiento de cinco especies de leguminosas como cobertura viva en palma aceitera en el estado Monagas, Venezuela
}

\author{
Behavior of five species of legume as cover crop \\ in oil palm in Monagas State, Venezuela \\ Renny Barrios-Maestre ${ }^{1}$, José Fariñas, Ramón Silva-Acuña, Damelys Sanabria
}

\section{RESUMEN}

Tradicionalmente para plantaciones de palma aceitera se ha utilizado la leguminosa Pueraria phaseoloides como cobertura viva, a fin de favorecer la fijación biológica del nitrógeno, controlar malezas, aportar materia orgánica y reducir la erosión. Cuando esta leguminosa se desarrolla compite con el cultivo y la cobertura pierde su efectividad. Este estudio se realizó en el Campo Experimental del Instituto Nacional de Investigaciones Agrícolas Monagas, en un lote sembrado con palma aceitera de seis meses de edad, del material genético Deli x Mobai. Se evaluaron cinco especies de leguminosas promisorias como coberturas vivas: Stylosanthes capitata CIAT 10280, Centrosema rotundifolium CIAT 5260, Arachis pintoi CIAT 18748, Desmodium ovalifolium CIAT 23665 y Pueraria phaseoloides, en un diseño de bloques al azar con cuatro repeticiones. Se cuantificó la población por metro cuadrado, altura de la leguminosa, producción de materia seca a los 180 días después de la siembra y el índice de cobertura específico. Los resultados indicaron que el índice de cobertura fue favorecido por la mejora en las condiciones de humedad del suelo. P. phaseoloides presentó el más rápido establecimiento y compitió eficientemente con el complejo de malezas; de manera similar, A. pintoi mostró mejor crecimiento bajo condiciones de buena humedad del suelo y tendencia a mayor desarrollo en lugares con baja incidencia solar. S. capitata y $C$. rotundifolium presentaron baja capacidad competitiva contra las malezas. D. ovalifolium mostró un índice de cobertura elevado y estable aun bajo condiciones de estrés hídrico y una producción adecuada de biomasa aérea y representa la mejor alternativa de cobertura viva en palma aceitera bajo las condiciones locales.

Palabras clave: Manejo de suelos, índice de cobertura, control de malezas, Pueraria, Desmodium, Arachis.

\section{ABSTRACT}

For palm oil plantations, traditionally the legume Pueraria phaseoloides has been used as cover crop, to promote the biological fixation of nitrogen, to control weeds, to supply organic matter and to reduce the erosion. When this legume develops, it competes with the crop and the efficiency of cover is lost. This study was carried out in the Experimental Field of the Instituto Nacional de Investigaciones Agricolas Monagas, on a palm oil plantation of the genetic material Deli $\times$ Mobai aged six months. Four species of promissory legumes were evaluated as cover crop: Stylosanthes capitata CIAT 10280, Centrosema rotundifolium CIAT 5260, Arachis pintoi CIAT 18748, Desmodium ovalifolium CIAT 23665 were compared with Pueraria phaseoloides, in a design of random blocks with four repetitions. Per square meter, the height of the legume, dry matter production at 180 days after sowing and a cover index of soil were quantified. The results indicate that the cover index was improved by soil humidity. P. phaseoloides presented the fastest establishment and competed efficiently with the complex of weeds. A. pintoi showed better growth under conditions of good soil humidity and tended to develop better in places with low solar incidence. S. capitata and C. rotundifolium presented low competitive capacity against the weeds. D. ovalifolium showed a high and stable cover index still under conditions of water stress and represents the best alternative of cover crop in oil palm under the local conditions.

Key words: Soil management, cover index, weed control, Pueraria, Desmodium, Arachis.

\section{Introducción}

El recurso suelo puede degradarse si es utilizado de manera intensiva con cultivos perennes $\mathrm{y}$, como consecuencia, se propende a la pérdida de productividad y calidad de la producción; bajo esta condición, la palma aceitera por ser un cultivo perenne requiere de técnicas de manejo de suelos que permitan mantener la productividad en el tiempo.

En plantaciones de palma aceitera, en especial aquellas establecidas en suelos susceptibles a la degradación, pobres en materia orgánica, fósforo,

1 Instituto Nacional de Investigaciones Agrícolas. San Agustín de La Pica, Vía Laguna Grande. Apdo. Postal 184. Telef. + 58 291 6413349. Maturín, Monagas. Venezuela. E-mail: rbarrios@inia.gob.ve 
potasio, magnesio y calcio, como son los suelos de Monagas (Salas, 1992), una de las alternativas para su manejo sostenible es utilizar especies de leguminosas como coberturas vivas. Las coberturas de leguminosas se usan de muchas maneras para la conservación y el mejoramiento de la productividad del suelo y son utilizadas en plantaciones de palma aceitera para fijar nitrógeno atmosférico; actúan además mejorando la aireación del suelo, la capacidad de retención y movimiento del agua, permiten en el suelo la incorporación rápida de grandes cantidades de materia orgánica y como consecuencia se reducen las variaciones de temperatura del suelo (ASD Costa Rica, 2001). Particular importancia poseen estos bancos de proteínas para la cría y engorde de una ganadería de menor porte.

Las coberturas de leguminosas desempeñan un importante papel de resistencia en el proceso de erosión, pudiendo retardarlo en una proporción de 10.000 veces (Páez, 1999). La acumulación de desechos foliares en los cultivos de leguminosas puede comenzar alrededor de seis meses después del establecimiento de la cobertura y los retornos de nitrógeno al suelo, que son bajos durante el primer año de desarrollo, se elevan a partir del segundo año cuando se constatan elevados tenores de nitrógeno (Zacaria, 1998).

La siembra de coberturas de leguminosas se ha convertido en una práctica común en el manejo de plantaciones de palma aceitera. Jalani y Ariffind (1998) y Barrios et al. (2000) agregan que dentro de la familia de las leguminosas existen varias especies que son comúnmente utilizadas como cobertura viva en plantaciones de palma aceitera. La selección de una especie depende de su adaptabilidad, comportamiento en las condiciones ecológicas de la zona a establecerse y del efecto que tenga sobre el cultivo.

En Venezuela pocas investigaciones se han realizado en este aspecto (Barrios et al., 2000) y solo se ha utilizado Pueraria phaseoloides en grandes extensiones, pero esta leguminosa una vez desarrollada afecta a la palma aceitera por su hábito de crecimiento trepador y pierde su eficiencia por efecto del sombreamiento que genera el dosel de la palma adulta.

Por lo antes expuesto, el objetivo de este estudio es el de evaluar leguminosas como coberturas vivas, al comparar el comportamiento de cinco accesiones, cuatro de ellas pertenecientes a la colección del CIAT con relación al de Pueraria phaseoloides, que viene siendo utilizada como cobertura de forma tradicional en palma aceitera.

\section{Materiales y Métodos}

\section{Ubicación y características del área experimental}

El estudio se realizó en el Campo Experimental del Centro de Investigaciones Agrícolas del estado Monagas (CIAE Monagas), ubicado en San Agustín de la Pica, a $18 \mathrm{~km}$, al noreste de Maturín (953'35" $\mathrm{N}, 62^{\circ} 59^{\prime} 30^{\prime}$ ' O, a 20 msnm), sobre una plantación de palma aceitera con seis meses de establecimiento del material genético Deli $\times$ Mobai. La zona presenta un clima ligeramente húmedo y cálido, con temperaturas promedio de $28{ }^{\circ} \mathrm{C}$, precipitación promedio anual de $1.300 \mathrm{~mm}$ y humedad relativa superior a 80\% (MARNR, 1997). La vegetación original correspondía al bosque húmedo tropical, pero ha sido sustituida casi completamente con fines agropecuarios.

La unidad de suelo bajo estudio corresponde a un Typic Plinthaqult, franca fina, silícica, isohipertérmica. Presenta problemas localizados de inundación por agua de lluvias, la cual puede durar sobre el terreno alrededor de cuatro a cinco días, el drenaje interno es lento y existe evidencia de mesa de agua estacional ocurriendo, en promedio, a unos $80 \mathrm{~cm}$ de profundidad (Luque y Rodríguez, 1977). El suelo del área experimental es franco-arenoso, ácido ( $\mathrm{pH} 4,5)$, con $1,72 \%$ de materia orgánica y de baja fertilidad natural. Los valores de fósforo, potasio, calcio, magnesio y zinc fueron 13,6, 95, 32 y 19 ppm, respectivamente, y los de cobre, zinc, hierro y manganeso: $0,56,0,5,62$ y $0,8 \mathrm{ppm}$ respectivamente.

El complejo de malezas estaba constituido por gramíneas y ciperáceas como: Paspalum convexum, Paspalum virgatum, Panicum maximum, Axonopus sp., Cyperus flavus, Cyperus sp., Ludwigia erecta, Ludwigia octovalvis, Sida rhombifolia, Sida sp., Indigofera sp., entre otras.

\section{Aspectos climáticos}

La información climatológica de precipitación y evaporación se obtuvo de la Estación Meteorológica del INIA Monagas. El cálculo del balance hídrico se realizó en forma acumulativa para periodos de cinco días. 


\section{Preparación del suelo, fertilización y manejo de las semillas}

Antes de la siembra de las especies de leguminosas se efectuaron tres pases de rastra. Se aplicó una fertilización básica de $500 \mathrm{~kg} / \mathrm{ha}$ de fosforita ( $28 \%$ de $\mathrm{P}_{2} \mathrm{O}_{5}, 21 \%$ de $\mathrm{CaO}$ y $4 \%$ de $\mathrm{S}$ ), la cual fue incorporada al suelo con el último pase de rastra. A los 30 días después de la siembra (dds) se reabonó con 100,60 y $50 \mathrm{~kg} / \mathrm{ha}$ de fosforita, cloruro de potasio y sulfato de magnesio, respectivamente.

Las semillas de las leguminosas fueron suministradas por la Unidad de Recursos Genéticos del Centro Internacional de Agricultura Tropical (CIAT). Se utilizó material vegetativo para la siembra de Arachis pintoi en hileras separadas a $50 \mathrm{~cm}$ y a $50 \mathrm{~cm}$ entre plantas. Las otras especies de leguminosas fueron sembradas con semillas sexuales distribuidas a chorro corrido, a una profundidad de siembra de $3 \mathrm{~cm}$ y en hileras separadas de $50 \mathrm{~cm}$.

Sólo las semillas de Stylosanthes capitata CIAT 10280 fueron escarificadas con ácido sulfúrico al 96-98\% (v/v) durante 10 minutos. No se aplicó herbicida preemergente y el control posemergente de malezas se realizó de forma manual a los 45 días después de la siembra (dds) de las leguminosas.

\section{Tratamientos y diseño experimental}

Los tratamientos estuvieron constituidos por las especies y su correspondiente accesión, distribuidos de la siguiente manera: tratamiento 1) Pueraria phaseoloides (testigo local); tratamiento 2) Stylosanthes capitata CIAT 10280; tratamiento 3) Centrosema rotundifolium CIAT 5260; tratamiento 4) Arachis pintoi CIAT 18748 y tratamiento 5) Desmodium ovalifolium CIAT 23665. Estas accesiones fueron seleccionadas entre 11 previamente evaluadas por Barrios et al. (2000, 2004) debido a su adaptación a la zona de estudio y por su hábito no voluble. En el Cuadro 1 se indica el hábito de crecimiento, longevidad y forma de propagación de las especies evaluadas.

El diseño estadístico empleado fue bloques al azar con cinco tratamientos, cuatro repeticiones, para un total de 20 unidades experimentales de $324 \mathrm{~m}^{2}(36 \mathrm{~m} \times 9 \mathrm{~m})$. Las evaluaciones se realizaron mensualmente durante seis meses, cuantificándose: altura de la leguminosa $(\mathrm{cm})$, porcentaje de cobertura de la leguminosa y de la maleza, y población de la leguminosa (plantas $/ \mathrm{m}^{2}$ ) . La altura fue medida desde la superficie del suelo con una vara graduada. La cobertura fue determinada visualmente y la población de leguminosas cultivadas contando el número de individuos en cuadrículas de $50 \mathrm{~cm} \times 50 \mathrm{~cm}$. Se determinó el Índice de Cobertura Específica de las leguminosas evaluadas, definido como el producto del porcentaje de cobertura del suelo por el porcentaje de cobertura de la leguminosa, dividido entre 100. Los valores fueron promediados para fines de análisis.

A los seis meses después de la siembra (mds) se determinó la biomasa aérea de leguminosas y del complejo de malezas, cortando las plantas manualmente al ras del suelo. Se tomaron cinco muestras al azar de $2.500 \mathrm{~cm}^{2}$ cada una $(50 \mathrm{~cm} \times 50 \mathrm{~cm})$ por cada unidad experimental, para un total de 100 muestras. Las muestras fueron secadas en estufa a $60 \pm 1{ }^{\circ} \mathrm{C}$ hasta peso constante. Finalmente se separaron las fracciones de leguminosas y malezas, y luego se pesaron en una balanza digital con precisión de $0,1 \mathrm{~g}$.

\section{Resultados y Discusión}

\section{Altura de la leguminosa}

Por medio del análisis de varianza se detectaron diferencias estadísticas para las especies, así como también para las fechas de evaluación y la interacción especies $\times$ fechas de evaluación. Al analizar el comportamiento de la altura de las leguminosas en el

Cuadro 1. Hábito, longevidad y forma de propagación de cinco leguminosas evaluadas como coberturas vivas en plantaciones de palma aceitera.

\begin{tabular}{lccc}
\hline Tratamientos & Hábito & Longevidad & Propagación \\
\hline 1. Pueraria phaseoloides & Hierba voluble & Perenne & Semilla \\
2. Stylosanthes capitata CIAT 10280 & Hierba erecta & Bianual & Semilla \\
3. Centrosema rotundifolium CIAT 5260 & Hierba rastrera & Perenne & Semilla \\
4. Arachis pintoi CIAT 18748 & Hierba rastrera & Perenne & Estolón \\
5. Desmodium ovalifolium CIAT 23665 & Hierba erecta & Perenne & Semilla \\
\hline
\end{tabular}


tiempo (Figura 1), se observa que varía dependiendo del hábito de crecimiento de la leguminosa y de la disponibilidad de humedad en el suelo.

$\mathrm{El}$ aumento en la altura de las leguminosas se produjo cuando las condiciones de humedad mejoran en el suelo, destacando las especies $P$. phaseoloides, $S$. capitata CIAT 10280, D. ovalifolium CIAT 23665 , todas con alturas superiores a 45 centímetros. Asimismo, P. phaseoloides presentó la mayor altura en las etapas iniciales de desarrollo, pero las accesiones representadas por las especies $D$. ovalifolium lograron superar en altura a esta especie cuando el déficit hídrico disminuye.

En la Figura 1 puede observarse que entre enero y abril cada especie mantiene una altura relativamente estable, lo cual probablemente se debe a las escasas precipitaciones ocurridas en ese lapso. Entre mayo y junio la altura tiende a incrementarse, particularmente para Stylosanthes capitata y Desmodium ovalifolium, las cuales son hierbas erectas, que en condiciones apropiadas predomina el crecimiento vertical, superando los $50 \mathrm{~cm}$. $P$. phaseoloides también incrementó su altura por encima de los $40 \mathrm{~cm}$, mientras que $C$. rotundifolium y A. pintoi se mantuvieron por debajo de los $20 \mathrm{~cm}$, lo cual es característico de ambas especies. En A. pintoi se producen ramas erectas cortas y ramas rastreras a partir de las yemas axilares, mientras que en $C$. rotundifolium casi todas las yemas axilares originan ramas rastreras que crecen al ras del suelo.
El hábito voluble de $P$. phaseoloides constituye una desventaja para las palmas jóvenes, ya que pueden quedar completamente cubiertas por esta leguminosa, compitiendo por espacio, luz y nutrimentos. Esto retrasa el crecimiento del cultivo y encarece el mantenimiento de las plantaciones en establecimiento.

Por otro lado, al estudiar el hábito de crecimiento de tipo enredadera y la altura que puede alcanzar $P$. phaseoloides se observa que constituye una desventaja para las palmas jóvenes, ya que esta especie puede trepar y cubrir cien por ciento al cultivo, interrumpiendo su desarrollo. D. ovalifolium, aunque puede superar en altura al resto de los materiales evaluados no afecta negativamente a las palmas por su hábito de crecimiento de tipo rastrero. $S$. capitata, con un hábito de crecimiento erecto, fue la especie que presentó la mayor altura; existe una respuesta positiva en crecimiento de la planta cuando mejora la humedad en el suelo, logrando alturas alrededor de 60 centímetros. A. pintoi y C. rotundifolium presentaron la menor altura, llegando alcanzar alrededor de 20 centímetros, debido a su hábito de crecimiento de tipo rastrero.

Arzola et al. (2006) encontraron la tendencia de $A$. pintoi a superar en altura a $P$. phaseoloides, lo cual atribuyeron al hábito de crecimiento de las plantas y a que $A$. pintoi se plantó con material vegetativo, lo que favoreció su establecimiento inicial, en comparación con $P$. phaseoloides, que

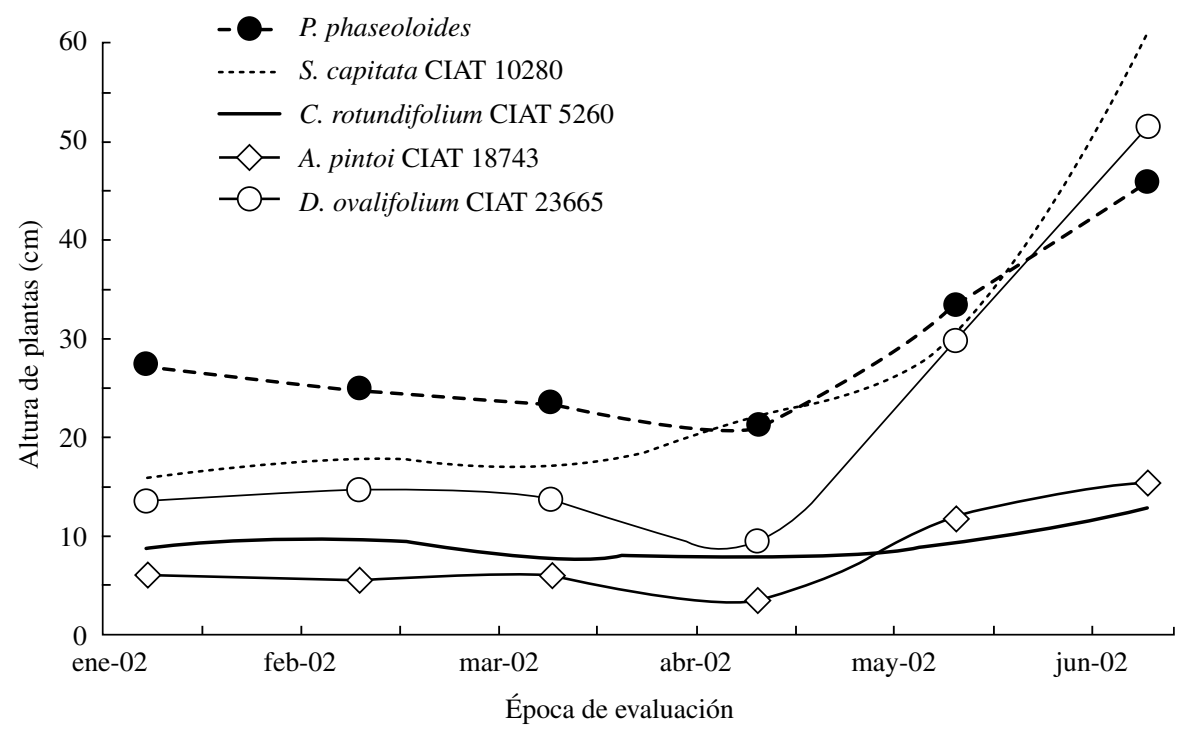

Figura 1. Altura de cinco especies de leguminosas utilizadas como cobertura viva en plantaciones de palma aceitera en el Estado Monagas. 
se sembró con semilla botánica. A pesar de que en este trabajo la siembra de semillas se realizó de la misma manera a la señalada por estos investigadores, los resultados fueron totalmente opuestos.

\section{Índice de cobertura específica}

El análisis de varianza mostró diferencias significativas para las especies, para las fechas y para la interacción especies $\times$ fechas de evaluación. La Figura 2 muestra las variaciones del índice de cobertura específico de las cinco especies de leguminosas y su relación con el balance hídrico acumulativo por períodos de cinco días, a fin de comparar las especies durante épocas diferentes.

El índice de cobertura del suelo presentó un comportamiento diferente para todas las accesiones, dependiendo de la condición hídrica del suelo. En todos los casos existe una respuesta positiva de las especies de leguminosas cuando la disponibilidad hídrica es adecuada, mientras que en condiciones de estrés, bien sea por déficit o por exceso de humedad, existe una actuación diferencial de las especies, donde unas muestran más susceptibilidad que otras.

P. phaseoloides presentó el más rápido crecimiento y la mayor cobertura inicial del suelo cuando es comparada con el resto de las leguminosas, logrando alcanzar un índice de cobertura superior al $70 \%$ a los 90 días después de la siembra (dds), lo que indica su particular capacidad de establecimiento inicial; sin embargo, mostró susceptibilidad al déficit hídrico (febrero-abril y septiembreoctubre), lo que trajo como consecuencia pérdida de hojas como mecanismo de defensa, se produjo disminución en la cobertura del suelo ubicándose alrededor de $40 \%$. Igualmente, bajo condiciones de exceso de humedad (junio-agosto) también se constató disminución en la cobertura, aunque no tan marcada como en el caso de déficit, lo cual demuestra una mejor tolerancia a las inundaciones temporales.

Una vez desarrollada, $P$. phaseoloides afectó negativamente al cultivo. Schultze-Kraft y Schmidt (1998) señalan que esta leguminosa presenta hábito de crecimiento de tipo enredadera y muy vigoroso, constituyendo problemas al cultivo principal si no existe un buen manejo. Sistema de Información de los Recursos del Pienso (2002) señala que esta especie presenta raíces profundas y no sufre por las sequías de corta duración.
D. ovalifolium presentó el mejor comportamiento en cuanto a índice de cobertura del suelo y habilidad competitiva con el complejo de malezas, incluso bajo condiciones de estrés. Durante todo el período de evaluación se observó una tendencia de la curva en forma ascendente, llegando a superar al kudzú tropical en algunas ocasiones. A diferencia de las otras especies, la pérdida de follaje no fue notoria, conservando el color verde de sus hojas en todo el período de evaluación.

Experiencias obtenidas por Schultze-Kraft y Schmidt (1998) señalan que el crecimiento de $D$. ovalifolium es vigoroso y muy agresivo, y posee excelente potencial de adaptación a diferentes condiciones de suelo y clima.

El comportamiento de $A$. pintoi demuestra que la principal dificultad que presenta esta leguminosa es su lento establecimiento y crecimiento inicial, además de poseer alta sensibilidad al déficit hídrico. De la Cruz et al. (1993) señalan que A. pintoi requiere buena humedad durante el establecimiento inicial en el campo y recomiendan su siembra al inicio de la época lluviosa y necesita, una vez establecida, alrededor de 4 a 6 meses para lograr una cobertura densa y uniforme. Los resultados obtenidos demuestran un índice de cobertura del suelo inferior al $20 \%$ y una clorosis de las hojas cuando no hay suficiente humedad en el suelo durante el periodo de establecimiento. Al disminuir el estrés hídrico, se obtuvo una respuesta rápida de la planta y alcanzó un índice de cobertura del suelo superior a $65 \%$ y competió en forma eficiente con las malezas de la zona de estudio, lo cual demuestra poca susceptibilidad a los excesos de humedad y mantiene una cobertura uniforme durante el resto del periodo de evaluación.

Trabajos de Argel y Pizarro (1992) señalan el uso del A. pintoi como una alternativa importante para el cultivo del café. La utilización de esta especie como cobertura puede contribuir a utilizar menos herbicidas, lograr mayor protección del suelo y el agua en zonas de ladera en donde se cultiva el café y a hacer un uso más eficiente de fertilizantes.

La especie $S$. capitata presentó dificultades durante el periodo de establecimiento. El hábito de crecimiento de tipo erecto de esta especie le confiere poca capacidad de cobertura y competencia con las malezas. Flores y Rodríguez (2002) señalan que esta leguminosa es tolerante a la sequía una vez desarrollada en el campo. Cuando disminuyó el déficit hídrico esta especie alcanzó un índice de 


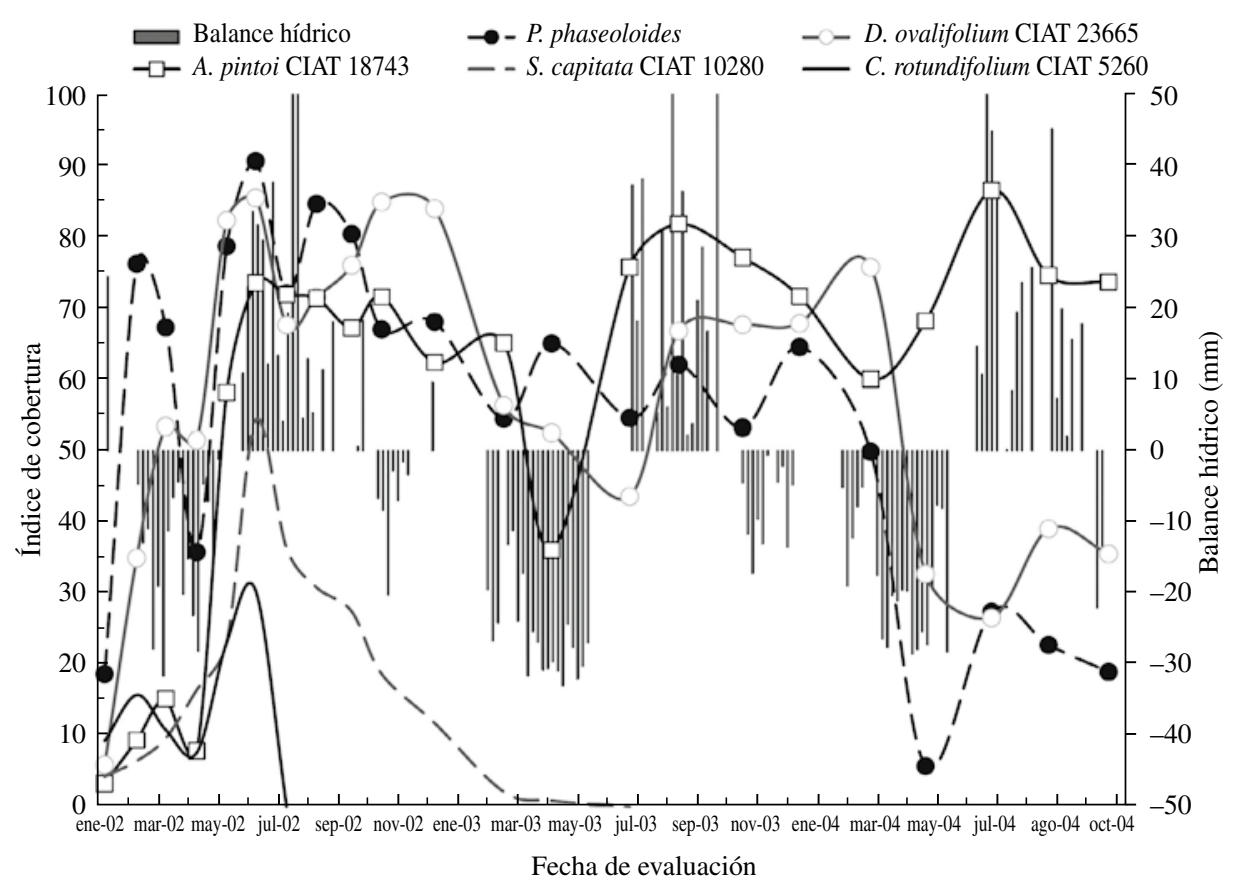

Figura 2. Variación del índice de cobertura específica de cinco leguminosas evaluadas como cobertura viva en plantaciones de palma aceitera.

cobertura alrededor de $50 \%$ y logró competir con las malezas; sin embargo, el exceso de humedad del suelo también afectó negativamente el índice de cobertura, mostrándose una disminución continua a partir del periodo de encharcamiento, hasta producirse su desaparición debido al cumplimiento de su ciclo bianual y a la escasa regeneración de la población por germinación de nuevas semillas.

En el caso de $C$. rotundifolium se demostró por su comportamiento que la planta presenta mucha sensibilidad al estrés hídrico, aún así, logró alcanzar un índice de cobertura alrededor de $25 \%$ durante el periodo de establecimiento, bajo condiciones de déficit hídrico. Cuando el déficit hídrico se redujo, esta especie no logró aumentar la cobertura del suelo. Esto puede deberse al hábito de crecimiento tipo rastrero que no le permite competir con el complejo de malezas y así poder lograr una buena cobertura en el suelo. En condiciones de exceso de humedad, C. rotundifolium mostró alta susceptibilidad que se reflejó prácticamente en la desaparición de la especie como consecuencia de los periodos de encharcamiento por la asfixia de las plantas adultas y de las semillas.

Este comportamiento contrasta con lo señalado por Barrios et al. (2004), quienes presentaron a
C. rotundifolium como una especie promisoria como cultivo de cobertura en suelos bien drenados.

\section{Población de leguminosas}

El análisis de varianza para el número de plantas por metro cuadrado arrojó diferencias significativas para las especies, para las fechas de evaluación y para la interacción especies $\times$ fechas de evaluación.

Para efectos del desdoblamiento de la interacción se construyó Figura 3, en la cual se demuestra que para todos los casos existe un control natural de las poblaciones en la medida que prevalece mayor crecimiento de las especies, expresada a través de la reducción del número de plantas en el tiempo. Este control se realiza para regular el uso de agua, nutrientes, luz y espacio físico.

Las leguminosas que mostraron mayor número de plantas por metro cuadrado fueron Pueraria phaseoloides y Desmodium ovalifolium CIAT 28665, destacando en todos los casos la primera especie; mientras que las accesiones Stylosanthes capitata CIAT 10280, Arachis pintoi, CIAT 18743 y Centrosema rotundifolium CIAT 5260 presentaron la menor población por metro cuadrado. 


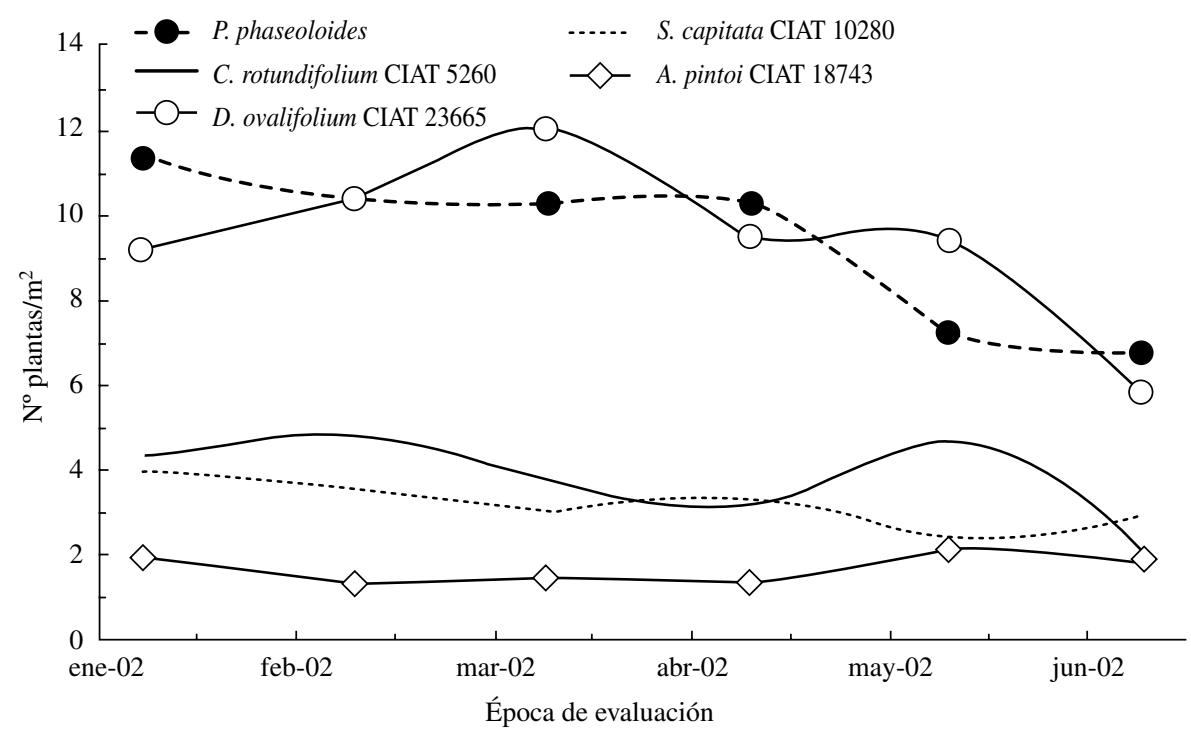

Figura 3. Comportamiento de la población por metro cuadrado de cinco especies de leguminosas evaluadas como cobertura viva en plantaciones de palma aceitera en el estado Monagas.

La reducción de la población fue más marcada en las especies $P$. phaseoloides y D. ovalifolium, lo cual se atribuyó a la intensidad de competencia y a las condiciones climáticas. Trabajos de Arzola et al. (2006) encontraron reducción en la población de $P$. phaseoloides debido a la pudrición del material de siembra, como consecuencia del encharcamiento en el suelo. Por su parte, Flores (1983) encontró que la alta precipitación limita el desarrollo de $P$. phaseoloides.

\section{Producción de materia seca a los 180 días después de la siembra}

De manera general, en la comparación de los promedios de la materia seca de las especies evaluadas y de las malezas (Cuadro 2) se puede constatar que a medida que disminuye la materia seca de la leguminosa se incrementa la de maleza. La mayor producción de materia seca se observó en D. ovalifolium y P. phaseoloides y sus correspondientes menores producciones de materia seca en las malezas. En la mayoría de los casos existe mayor biomasa aérea de la leguminosa que de las especies de malezas, con excepción de $C$. rotundifolium.

Para las leguminosas $P$. phaseoloides y D. ovalifolium se evidencia el mayor contenido de biomasa aérea, cuando son comparadas con el resto de los materiales, con valores ubicados alrededor de 80 gramos. Ambas presentaron un comportamiento muy agresivo para competir con malezas; D. ovalifolium puede emitir en cada planta cinco extremidades alrededor de dos metros de largo que se extienden y entrelazan sobre la superficie del suelo y $P$. phaseoloides, debido a su hábito de crecimiento rastrero-enredadero, también limita el crecimiento y desarrollo de malezas.

Trabajos de Zwart et al. (2005) destacan la mayor producción de biomasa aérea de $D$. ovalifolium en comparación con A. pintoi y Centrosema molle, y señalan que la biomasa producida por estas leguminosas retorna y se incorpora al suelo por medio de la hojarasca que cae sobre el mismo o puede ser utilizada como alimentación animal gracias al rico contenido nutricional que estas poseen.

Las especies A. pintoi y $S$. capitata presentaron comportamientos muy similares en cuanto a biomasa aérea. La primera accesión mostró 45 gramos contra 35 gramos materia seca de malezas como promedio, mientras que $S$. capitata mostró alrededor de 32 gramos contra 28 gramos de biomasa aérea de malezas.

En el caso de $C$. rotundifolium se observó que no ejerce un control eficiente de malezas, el cual está influenciado por su hábito de crecimiento rastrero y su alta susceptibilidad al déficit hídrico. La relación biomasa aérea vs. biomasa de malezas fue de 1:3, esta condición denota el grado de deterioro 
Cuadro 2. Producción de materia seca a los 180 dds de cinco especies promisorias como cobertura viva en plantaciones de palma aceitera en el estado Monagas.

\begin{tabular}{llccc}
\hline Especie & \multicolumn{2}{c}{ Materia seca especie $\left(\mathrm{g} / \mathrm{m}^{2}\right)$} & \multicolumn{2}{c}{ Materia seca malezas $\left(\mathrm{g} / \mathrm{m}^{2}\right)$} \\
\hline D. ovalifolium CIAT 23665 & 355,7 & A & 46,8 & B \\
P. phaseoloides & 330,9 & A & 80,8 & B \\
A. pintoi CIAT 18748 & 160,3 & B & 11,8 & B \\
S. capitata CIAT 10280 & 130,4 & B & 110,1 & B \\
C. rotundifolium CIAT 5260 & 55,2 & C & 227,4 & A \\
\hline
\end{tabular}

de la materia seca de la leguminosa en relación al de las malezas que compiten por área, agua y luz. De manera similar, al analizar la estructura morfológica de $C$. rotundifolium se evidencian tallos muy delgados extendidos sobre la superficie del suelo que pueden llegar hasta 1 metro de largo en la última evaluación, a esto se agrega que las plantas no emiten ramificaciones erectas.

\section{Conclusiones}

La habilidad competitiva de las leguminosas con el complejo de malezas se incrementó para todas las especies de leguminosas cuando mejoraron las condiciones de humedad en el suelo, destacándose las especies $D$. ovalifolium y $P$. phaseoloides. A. pintoi mostró un comportamiento intermedio, mientras que $C$. rotundifolium y $S$. capitata presentaron la menor habilidad competitiva con las malezas, el menor índice de cobertura del suelo y menores aportes de materia seca.

Las especies $P$. phaseoloides y $D$. ovalifolium presentaron el mayor número de plantas por metro cuadrado, mientras que $A$. pintoi mostró la menor población por metro cuadrado. $C$. rotundifolium y $A$. pintoi tienen la posibilidad de renovar su población debido a la producción de un banco de semillas que germinan con adecuadas condiciones de humedad en el suelo.

P. phaseoloides posee rápido establecimiento, pero debido a su hábito trepador compite con las palmas jóvenes afectándolas negativamente; tolera inundaciones temporales y aporta grandes cantidades de materia seca; mientras que el establecimiento de $A$. pintoi fue muy lento y posee poca tolerancia al déficit hídrico, lo cual limita su uso en plantaciones jóvenes. La mayor producción de biomasa aérea la obtuvieron las especies $P$. phaseoloides y D. ovalifolium.

D. ovalifolium presentó el mejor comportamiento en índice de cobertura del suelo bajo condiciones de estrés hídrico, demostrando buena adaptabilidad a las condiciones locales. Representa una alternativa al kudzú tropical ( $P$. phaseoloides) como cobertura viva en palma aceitera.

\section{Literatura Citada}

Argel P. y Pizarro, E.

1992 Germplasm case study: Arachis pintoi. In Pastures for the tropical lowlands. Colombia, Centro Internacional de Agricultura Tropical. CIAT Publications (211): 57-73.

Arzola A.; Castillo E.; Valles B. y Jarillo, J.

2006 Establecimiento sin labranza de Arachis pintoi y Pueraria phaseoloides en pasturas nativas. Pasturas Tropicales 28 (3): 51-55.

ASD Costa Rica

2001 Coberturas en palma aceitera. En: Actualización en la producción de palma aceitera. Maturín (Venezuela). Mayo 2-4. 2001. Lecturas selectas. Maturín. pp. 90-94.

Barrios, R.; Molina, D.; Fariñas, J.; Barreto, F. y Matos, G.

2000 Avances en la evaluación de leguminosas promisorias como cobertura viva en palma aceitera. En: Experiencias en el género Centrosema de Venezuela. Anzoátegui (Venezuela). Nov. 27-29. Simposio. Anzoátegui. pp. 87-92.
Barrios R.; Fariñas J.; Díaz A. y Barreto, F

2004 Evaluación de once accesiones de leguminosas como coberturas vivas en palma aceitera en el estado Monagas, Venezuela. Bioagro 16 (2): 113-119.

De la Cruz, R.; Suárez, S. y Ferguson, J.

1993 The contribution of Arachis pintoi as a ground cover in some farming systems of Tropical America. In: Biology and Agronomy of Forage Arachis pintoi. Colombia, CIAT. pp. 102-108.

Flores, M.

1983 Bromatología animal, 3a. ed. Limusa, México, D.F.

Flores, A y Rodríguez, I.

2002 La alfalfa criolla: alternativa forrajera para los ganaderos de la Mesa de Guanipa. Fonaiap Divulga Número 60: 28-32. Jalani, B y Ariffind, D.

1998 Leguminosas y Cobertura Vegetal en plantaciones de palma aceitera en el Sudeste de Asia. En Seminario Internacional 
Cobertura de Leguminosas en Cultivos permanentes. Santa Bárbara del Zulia (Venezuela). Oct. 1-2. 1998. Compendio. Zulia. pp. 83-93.

Luque, O. y Rodríguez, T.

1977 Estudio agrológico detallado: Campo Experimental del Centro de Investigaciones Agropecuarias Región Nororiental (La Pica-estado Monagas). Boletín técnico (1): 64.

MARNR

1997 Atlas del Estado Monagas. Ed. Gobernación del Estado Monagas. 99 pp.

Páez, M.L.

1999 Degradación del suelo. La erosión hídrica. En: Conservación de suelos y aguas. Maracay, Universidad Central de Venezuela. Facultad de Agronomía. Mimeografiado. pp. 22-47.
Schultze-Kraft, R. y Schmidt, A.

1998 Experiencias de leguminosas de cobertura. En: Seminario Internacional Cobertura de Leguminosas en Cultivos Permanentes. Santa Bárbara del Zulia (Venezuela). Oct. 1-2. 1998. Compendio. Zulia. pp. 29-44.

Salas, R.

1992 Palma aceitera. Caracas, Venezuela. Universidad Central de Venezuela. 150 p.

Zacaria, Z.

1998 Manejo de suelos y fertilizantes en plantaciones de palma aceitera en Malasia. Palmas (Colombia) 19, Número especial: 207-215.

Zwart, M.A.; Rojo, J.M.; De la Cruz, R. y Yeomans, J.

2005 Coberturas y la salud del suelo. Tierra Tropical (Costa Rica) (2005) 1 (1): 9-20. 